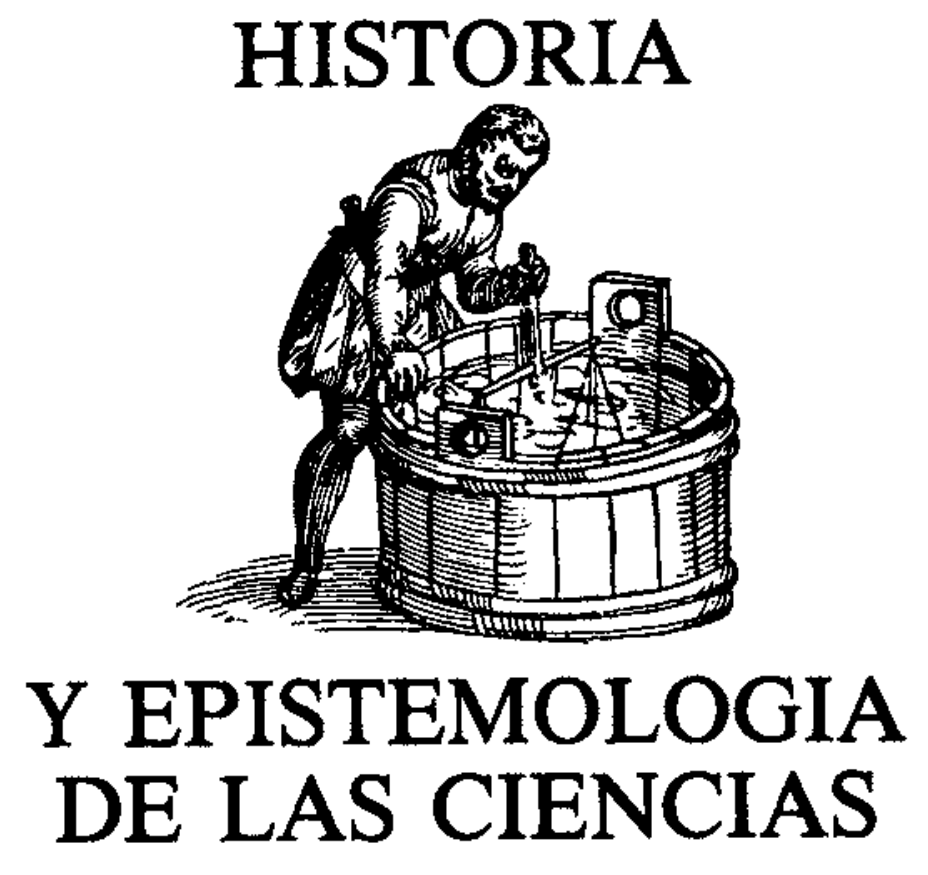

\title{
LA ENSEÑNZA DE "FUERZA Y MOVIMIENTO" COMO CAMBIO CONCEPTUAL
}

HEWSON, P.W.
Departamento de Curriculum e Instrucción Universidad de Wiscosin-Madison Madison, WI 53706

Versión castellana de Joaquín Martínez Torregrosa

\section{SUMMARY}

Research has shown that many students hold alternative conceptions about motion and the factors which influence it and that an important component of these conceptions are epistemological commitments, one example of which is a cause-effect relationship. The article describes a series of microcomputer programs designed to facilitate conceptual change from an impetus-type view to a Newtonian view. A significant feature of these programs is the explicit focus given to the nature of the relationship between cause and effect and its role in the conceptual change process.

\section{INTRODUCCIÓN}

Durante los últimos años se han publicado muchos estudios de las concepciones de los estudiantes sobre movimiento y los factores que influyen en él. Dichos estudios suministran una amplia evidencia de que los 
estudiantes llevan a la clase un conjunto diverso de ideas que no les han sido enseñadas. También hay evidencia de que estas ideas no parecen afectar en gran medida a la enseñanza, incluso cuando entran directamente en contradicción con el contenido newtoniano que ha sido enseñado. Puesto que los profesores quieren que los estudiantes aprendan lo que ellos enseñan, estos resultados señalan la necesidad de ayudar a que los estudiantes cambien sus creencias sobre el movimiento y los factores que influyen en él. La pregunta general es por tanto: $i$ Cómo y por qué cambian las personas sus opiniones?

Una manera de abordar esta cuestión viene sugerida por el modelo de aprendizaje como cambio conceptual (Posner et al. 1982, Hewson 1981). El modelo supone que probablemente las personas tienen buenas razones para mantener las ideas que tienen. Esto sugiere que es necesario responder a las siguientes preguntas: 1. ¿Qué concepciones no newtonianas mantienen los estudiantes sobre el movimiento y sus causas? 2 . ¿Por qué no ven los estudiantes los inconvenientes de estas concepciones? 3. ¿Por qué no ven las ventajas de las concepciones newtonianas? Conocer estas respuestas nos permitirá una crítica fundada de la enseñanza ya realizada, y suministrará una base para diseñar una enseñanza que se dirija específicamente a las concepciones de los estudiantes sobre el movimiento de los estudiantes. Así pues, es necesario considerar también las siguientes cuestiones: 4 . ¿Cuáles son Ias características de la enseñanza que ha tenido en cuenta las concepciones de los estudiantes? 5. ¿Qué deberían hacer -si es que algo hay que hacer- Ios profesores con las concepciones de los estudiantes en su enseñanza?

La sección II es una revisión de la literatura sobre las concepciones de los estudiantes sobre fuerza y movimiento, y la sección III considera los efectos de la enseñanza que tiene en cuenta estas concepciones, y de la que no los tiene. El propósito de estas secciones es responder las cuatro primeras preguntas de arriba.

La sección IV del artículo se centra en una serie de programas de ordenador sobre fuerza y movimiento, cuyo diseño estuvo guiado por el modelo de cambio conceptual. El propósito del programa fue ayudar a que los estudiantes que tenían concepciones tipo-impetus cambiaran a las concepciones newtonianas de fuerza y movimiento. No obstante, los programas también tocaban otros aspectos identificados en la revisión bibliográfica. Distintos aspectos de los programas y su diseño se analizan y justifican en la sección $V$. Estas dos secciones constituyen, por tanto, un estudio de una posible respuesta a la cuestión 5 .

\section{CARACTERÍSTICAS NOTABLES DE LAS CONCEPCIONES DE LOS ESTUDIANTES}

Algunos autores han usado conceptos newtonianos para organizar sus discusiones sobre la literatura exis- tente; resaltan la revisión de McDermott (1984) de muchos estudios diferentes, y el desarrollo por Halloun y Hestenes (1985) de una taxonomía de las "concepciones de sentido común sobre el movimiento", basada en sus propios estudios. Viennot (1985) ha mostrado que hacer eso puede conducir a pasar por alto importantes aspectos de las concepciones de los estudiantes, y esta revisión está organizada teniendo esto en cuenta.

\section{A. Contenido específico}

Con respecto al contenido existe evidencia de que:

* Los estudiantes tienen concepciones relativamente indiferenciadas de movimiento, confundiendo frecuentemente los conceptos de posición y velocidad (Trowbridge y McDermott 1980), y velocidad y aceleración (Trowbridge y McDermott 1981, Johansson et al. 1985).

* Las concepciones de los estudiantes sobre fuerza a menudo incluyen una u otra de las características siguientes: factores relativamente indiferenciados que causan el movimiento, con una confusión frecuente de las ideas de fuerza, cantidad de movimiento y energía cinética (Viennot 1979, Watts 1983, Minstrell 1984); una inexistencia de tipos pasivos de fuerza, como las fuerzas de reacción (Minstrell 1982); y, una faita de un componente de interacción, con la fuerza asignada al objeto mismo (Viennot 1985).

* Cuando se les da a los estudiantes el movimiento de un objeto en circunstancias conocidas, muchas respuestas incluyen dos características relacionadas. Primera, muchos estudiantes se centran en la dirección del movimiento, como la característica cinemática más significativa. Segundo, muchas respuestas inciuyen una idea de "impetus" o "persistencia de una causa primera" que depende del contexto. Estos rasgos aparecen consistentemente a través de variantes diferentes de la misma cuestión básica: Dado un movimiento, encontrar su causa y usarla para explicar el movimiento pasado y/o predecir el movimiento futuro.

(I) Si se les pide a los estudiantes que expliquen por qué un objeto se ha movido tal como to ha hecho, muchos lo hacen usando uno o más factores "causantes", a los que generalmente llaman fuerzas (Halloun y Hestenes 1985, Johansson et al. 1985, Viennot 1979, Champagne et al. 1980, Watts y Zylberstajn 198 I, Clement 1982, Hewson 1984). Esta idea ha sido resumida por Clement (1982) como "el movimiento presupone una fuerza".

(II) Si se les pide a los estudiantes que predigan el movimiento futuro de un objeto, quitando todos los factores causantes obvios, predicen que el movimiento previo continuará, pero no indefinidamente (Clement 1982, McCloskey et al. 1980).

(III) $\mathrm{Si}$ se les pide que predigan el movimiento futuro de un objeto, y están presentes factores causantes obvios, surge una competencia entre el movimiento previo y estos factores, dependiendo el resultado de su tamaño relativo (Clement 1982, Caramazza et al. 1981, Whitaker 1983). Estos resultados son reminiscencias 
de la teoría medieval del impetus: al poner un cuerpo en movimiento se te imparte un impetus que gradualmente se disipa, bien espontáneamente o debido a otras razones.

* Cuando se les da a los estudiantes una fuerza que pueden controlar, y se les pide que la usen para producir un determinado movimiento, muchas respuestas se basan en una idea que di Sessa (1982) resumió como: "los objetos deben ir en la dirección en que son empujados" (White 1982, Lawson 1984). Estas respuestas se manifiestan ante variantes de una cuestión básica inversa (de la anterior): dada la causa, predecir el movimiento. Implícitamente desconsideran el movimiento anterior, e incluyen una idea de "no-persistencia de la causa primera", en contraste con la noción del impetus anterior.

* Los estudiantes usan diferentes nociones de fuerza en distintas circunstancias, es decir, sus respuestas son dependientes del contexto (Champagne et al. 1985). Viennot (1979) propuso un modelo que subraya las condiciones bajo las cuales se usan diferentes concepciones. Este resuitado está también implícito en los dos parágrafos previos, y es tratado más detalladamente por Hewson (1985).

Las características que acabamos de subrayar parecen ser independientes de las técnicas de identificación. Se han usado tareas de lápiz y papel por muchos autores. Di Sessa (1982) y White (1984) observaron cómo los estudiantes controlaban movimientos simulados por ordenador, y Lawson (1984) les pidió que demostraran su comprensión realizando tareas cualitativas en el laboratorio. La interpretacion de muchas de dichas tareas ha sido confirmada en entrevistas individuales con los estudiantes. Por último, Zietsman y Hewson (1986) no encontraron diferencia en el modo en que observaron el movimiento de objetos por simulación en el ordenador y objetos físicos en el laboratorio.

\section{B. Aspectos epistemológicos del contenido}

Diferentes autores han comentado el tema de la consistencia, o falta ella, en las respuestas de los estudiantes. Minstrell (1982) mostró claramente que hasta que no usaron "razonamiento consistente" en ejemplos diferentes, no fueron capaces de aceptar una explicación newtoniana de objetos en reposo. Whitaker (1983) identifico que respondían inconsistentemente, dando respuestas correctas a una o más -pero no a todascuestiones similares de cinemática. Halloun y Hestenes (1985) advirtieron un modelo de inconsistencia semejante respecto a un grupo de cuestiones similares de dinámica. McDermott (1984), al analizar las entrevistas de Lawson a estudiantes a quienes se pedía que controlaran el movimiento de discos de hielo seco en el laboratorio, comentó que carecían de un "esquema conceptual consistente" y usaban el término fuerza ambiguamente. Champagne, Gunstone y Klopfer (1985) advirtieron que, tanto antes como después de la instrucción, daban explicaciones que dependían de la situación específica de por qué las cosas se movían, y comentaron que parecían incapaces de la necesidad de consistencia a través de las distintas situaciones.

Hay evidencia de que las respuestas están también afectadas por su comprensión de la relación entre causa y efecto aplicados a fuerza y movimiento. Minstrell (1982) mostró que hasta que sus estudiantes no se fijaron en la simillitud de los efectos en Iugar de en las características de los objetos que causaban dichos efectos, las respuestas dependientes del contexto no se hicieron menos atractivas.

Hewson (1985) discutió el papel jugado por los presupuestos epistemológicos sobre la consistencia y generalizabilidad en un número de estudios diferentes, y mostró cómo se interaccionaban con las ideas de causa y efecto. Más específicamente, mostró que la inconsistencia está implícita en el modelo de Viennot de tres modos diferentes: el modeio suministra explicaciones del movimiento que son dependientes del contexto; permite dos tipos diferentes de pensar sobre el movimiento, dependiendo de si el movimiento es o no explícitamente conocido; y usa la palabra "fuerza" de dos maneras bastante diferentes. Todos estos aspectos son inconsistentes para dar lugar a una teoría. Con respecto a causa y efecto, el modelo de Viennot no incluye una relación simétrica, ya que predice respuestas distintas según se den las causas y se pidan los efectos, o según se den los efectos y se pidan las causas. A este respecto, al menos, el modelo de Viennot está de acuerdo con los resultados de contenido expuestos anteriormente. Hewson también analizo los resultados obtenidos por di Sessa (1982) y Lawson (1984) para mostrar que diferentes respuestas podrían ser explicadas cuando los estudiantes no usaban la consistencia y generalización en sus argumentos, aunque no fue posible decidir si no tenían esos presupuestos epistemológicos, o sí los tenían pero decidían no usarlos.

\section{Discusión}

Las investigaciones resumidas previamente apuntan hacia aspectos diferentes pero estrechamente interrelacionados. Está, por un lado, el contenido específico de las teorías de los estudiantes, y, por otro, la epistemología de dicho contenido, incluyendo su aplicación a un rango de ejemplos y su forma y estructura. El contenido específico reseñado arriba ha sído el objeto explícito de la mayor parte de la literatura publicada. En cambio, los aspectos epistemológicos implícitos en el contenido específico, han recibido muy poca atención. Por esta razón, el resto de esta sección se centrará en ellos. Creo que son la clave para comprender por qué frecuentemente parecen considerar las explicaciones tipo-impetus más atractivas que las ideas newtonianas.

Hay una ausencia de consciencia por parte de muchos estudiantes de la importancia de las características principales de una teoría científica, demostrada por el hecho de que frecuentemente no las usan. Con respecto a la precisión, muchos no diferencian entre términos teóricos tales como velocidad y aceleración, y fuerza, cantidad de movimiento y energía; estos términos se usan intercambiablemente. Por lo que se refiere a consis- 
tencia interna, muchos utilizan los mismos términos de formas cualitativamente diferentes, e. g., fuerza como la causa del movimiento y como lo que mantiene el movimiento. También, muchos estudiantes van de causas a efectos de manera distinta a como van de efectos a causas. Respecto a la generabizabilidad, muchos estudiantes no buscan las características comunes esenciales, sino que parecen usar rasgos superficiales para diferenciar sus explicaciones de hechos similares cualitativamente. Esto también puede interpretarse como una ausencia de parsimonia (prudencia, refiexión).

Una consecuencia del nulo uso de criterios tales como precisión, consistencia, generabizabilidad, y parsimonia al evaluar sus propias concepciones es que dichas concepciones resultan ser de utilidad limitada. Suministran explicaciones de hechos específicos, pero no incluyen criterios para determinar qué otros hechos pueden o no requerir explicaciones parecidas. A menudo son incapaces de predecir resultados de hechos de tipos similares, y dichos fallos es más probable que conduzcan a una proliferación de concepciones separadas que a un cambio hacia una concepción más general. En resumen, parece que los estudiantes que no usan dichos criterios son incapaces de apreciar los beneficios que proceden de las teorías que -como la mecánica newtoniana- los satisfacen.

Ésta puede ser entonces una razón de por qué muchos no aprenden mecánica newtoniana cuando se les enseña. Pero, ¿qué atractivo tienen las alternativas? Si se reemplazan la exigencia de predicción exacta y parsimoniosa, por explicaciones generalizables con criterios tales como naturalidad y proximidad a los fenómenos, está claro que las explicaciones tipo-impetus producen más sentido que las ideas newtonianas. Son más inteligibles; los estudiantes las comprenden más fácilmente que un complejo análisis newtoniano. Son más plausibles; es más fácil creer que una bicicleta gana velocidad porque se está pedaleando que porque la superficie de la carretera ejerce una fuerza hacia delante sobre la rueda trasera, o que una piedra que cae libremente es un movimiento más simple, más natural que el de un ciclista que mantiene su velocidad constante mientras pedalea entre un fuerte viento. En otras palabras, hay buenas razones para creer que, para los estudiantes sin un fuerte sentido de lo que una buena teoría puede hacer, los tipos de concepciones resumidas anteriormente son más fáciles de entender, tienen más probabilidad de ser vistas como de "sentido común" y no como algo menos útil que las concepciones newtonianas. En estas circunstancias, es mucho más fácil comprender por qué surgen y por qué son tan persistentes a pesar de una enseñanza en sentido opuesto.

\section{CONCEPCIONES DE LOS ESTU- DIANTES Y ENSENANZA}

\section{A. Revisión de estudios}

Cierto número de estudios ha considerado el efecto sobre las concepciones que tienen los estudiantes acerca de la instrucción convencional que no intenta tratar estas concepciones directamente (Halloun y Hestenes 1985, Champagne et al. 1980, Clement 1982, McCloskey et al. 1980, Caramazza et al. 1981). Aunque generalmente había una reducción en el número de respuestas no newtonianas, en todos los estudios una proporción substancial no cambió sus concepciones. Este resultado ha sido interpretado ampliamente como un fallo inaceptable de la instrucción convencional, y ha sido seguido generalmente por la recomendación de que las concepciones no newtonianas de los estudiantes debían ser enfrentadas. McClelland (1985) ha criticado este enfoque atribuyendo la presencia de estas concepciones a una instrucción pobre, y sugiere que pueden ser evitadas por una enseñanza más explícita, mejor. Los estudios anteriores no especificaron la "instrucción convencional" empleada, suponiendo que seguía los libros de texto estándard, y además que hacía justicia a la dinámica newtoniana.

Los estudios que asumen que las concepciones no newtonianas de los alumnos no pueden ser ignoradas han sugerido diferentes modos de tratarlos, y en ciertos casos han descrito los efectos de estas estrategias en los alumnos. En una serie de estudios basados en ordenador, fueron capaces de controlar el movimiento de un objeto en pantalla jugando a una secuencia de juegos que estaban diseñados para haceries entrar en conflicto con las inadecuaciones existentes en sus concepciones (di Sessa 1982, White 1984). En un estudio, de modo individual cambiaron sus estrategias mientras jugaban los juegos; y en otro, un grupo de estudiantes de escuela superior mostró mejoras significativas en sus respuestas a problemas de lápiz y papel sobre el control del objeto de pantalla después de jugar. No obstante, no se les preguntó cuestiones sobre movimiento en otros contextos.

Champagne, Gunstone y Klopfer (1985) describieron posibles estrategias de enseñanza que están basadas en la investigación en psicología cognoscitiva, y llevaron a cabo dos estudios en los que se usó una de estas estrategias -confrontación de ideas-. Aspectos que vale la pena resaitar de su desarrollo es que supone que los estudiantes exploren las implicaciones de sus concepciones mediante la predicción y el análisis, que comparen sus concepciones con las de otros, incluyendo el instructor, y la subsiguiente modificación de concepciones para afrontar inconsistencias y reconciliar diferencias. Esto se alcanzó dando una gran cantidad de tiempo a las discusiones en clase. Se registraron cam. bios conceptuales en las comprensiones de los alumnos de las "perspectivas científicas" del movimiento.

Minstrell $(1982,1984)$ describio las estrategias que usó en una clase de física de escuela superior para considerar por qué los objetos permanecen en reposo, aceleran uniformemente y se mueven a velocidad constante. Al principio, se animó a los estudiantes para que verbalizaran sus concepciones. Entonces se consideraron muchas experiencias diferentes relativas a estas ideas iniciales, y las observaciones fueron comparadas con las concepciones para resolver discrepancias. La discusión abierta jugó un papel importante en esta fase. 
Minstrell también suministró oportunidades repetidas para volver a utilizar argumentos en contextos diferentes, con ejemplos diferentes. Se registraron importantes cambios en la comprensión de los estudiantes hacia una explicación newtoniana de fuerza y movimiento.

Hewson $(1984,1985)$ utilizó estrategias basadas en un modelo de aprendizaje como cambio conceptual para diseñar un programa de ordenador referido a la concepción alternativa de velocidad de los alumnos. El modeIo de cambio conceptual (Posner et al. 1982, Hewson 1981) implica que la instrucción debería suponer la identificación, o diagnóstico, de las concepciones de los estudiantes, y la reducción del estatus de estas concepciones donde son alternativas a lo que se va a enseñar, junto con el aumento del estatus del nuevo contenido que se está enseñando. Cuando se aplicó a la velocidad, que muchos estudiantes confunden con posición, la prescripción del modelo de cambio conceptual pudo ser usada de una manera sencilia y dió lugar a cambios drásticos.

Halloun y Hestenes (1987) usaron ta teoría de la modelización para diseñar la enseñanza en resolución de problemas en mecánica elemental, y llevaron a cabo un experimento pedagógico para evaluar su efectividad. Su instrucción tuvo tres características principales: diseño sistemático y selección de problemas paradigmáticos para estudio intensivo, una estrategia dialéctica de enseñanza, y una introducción gradual de la teoría y técnicas de la modelización. La estrategia dialéctica de enseñanza contenía los siguientes elementos: formulación explícita de las creencias de sentido común, comprobar la consistencia con la evidencia empírica de dichas creencias, comprobar la consistencia interna mutua de dichas creencias, y comparación con las creencias alternativas. Se obtuvieron mayores puntuaciones en los exámenes de curso por diferentes grupos tratados, en comparacion con un grupo de control.

Hake (1987) utilizó una variedad de innovaciones instructivas en un curso de verano de mecánica elemental. Estas innovaciones incluían: laboratorios estructurados para inducir diálogos socráticos, conferencias fomentando un enfoque cualitativo a la resolución de problemas y contrastando los conceptos newtonianos y no newtonianos de los estudiantes sobre movimiento, y cintas de vídeo de la serie El Mundo Mecánico. Las sesiones de laboratorio fomentaban experiencias manuales para promover la formación de conceptos mediante "desequilibrio", discusión y diálogo socrático. Las puntuaciones en los exámenes precurso y postcurso de mecánica, sugirieron que el cruce de los estudiantes al mundo newtoniano había ocurrido.

Aspectos importantes de estas estrategias parecen incluir la realización de actividades que permiten que los estudiantes:

\section{Sean expuestos a un número de diferentes ejemplos.}

Identifiquen y planteen sus concepciones actuales verbalmente o respondiendo a un movimiento simulado o en alguna otra manera.
* Exploren las implicaciones de sus concepciones en una variedad de ejemplos requiriéndoles explicaciones autoconsistentes y predicciones.

* Adviertan las limitaciones de las concepciones que
tienen en ese momento.

\section{B. Discusión}

La revision de las concepciones de los estudiantes ha mostrado que las características notables eran tanto del contenido específico como de la epistemología de dicho contenido. Aunque la epistemología no ha sido explícitamente abordada en los estudios de enseñanza mencionados, las actividades propuestas requerían que abordaran estos aspectos. El uso de ejemplos diferentes que se comparan y contrastan mutuamente, para los que se busca una explicación consistente, y en los que se formulan y comprueban predicciones, está demandando consistencia y generalizabilidad. Por ejemplo, los juegos de ordenador de di Sessa y White requieren un flujo constante de predicciones basadas en ejemplos previos. En los estudios de Champagne et al., y de Halloun y Hestenes, se les pedía que compararan y contrastaran sus visiones con las del instructor, para afrontar las inconsistencias, y ser conscientes de la necesidad de reconciliar sus nociones con los conceptos y principios que se iban a aprender. En la estrategia de enseñanza de Minstrell y en el programa de Hewson sobre velocidad, se les incitaba a usar un ejemplo para pensar en el siguiente.

Las distintas metodologías usadas en estos trabajos indican que es posible alcanzar los objetivos anteriores de diferentes maneras. No es sorprendente que las discusiones de clase resalten con prominencia; da al profesor muchas oportunidades de responder inmediata y apropiadamente a las inquietudes y problemas del estudiante. No obstante, esto requiere que el profesor esté perfectamente preparado sobre todos los aspectos del contenido. Podría ser más sorprendente que programas de ordenador por sí solos fueran capaces de obtener los mismos resultados; esto, no obstante, depende de que el diseñador del programa fuera capaz de abordar los mismos aspectos que se requerían de los profesores de una manera flexible, explícita y premeditada.

Para que los estudiantes adviertan que las concepciones tipo-impetus que tienen necesitan ser cambiadas por las concepciones newtonianas, parece claro que necesitan comprender por qué las tienen. Estas razones parecen estar basadas en criterios epistemológicos de un buen "sentido común", pero que son incompatibles con una teoría científica precisa como la mecánica newtoniana. Si éste es el caso, es comprensible que la enseñanza que solamente trata Ios detalles específicos de los contenidos deseados tenga poca probabilidad de hacer impacto en las concepciones alternativas. Solamente cuando los estudiantes son capaces de plantear sus propias visiones explícitamente, comprenden las razones por las que son atractivas y comienzan a ver sus limitaciones, pueden apreciar las ventajas que emanan del uso de la mecánica newtoniana, una teoría científi- 
ca, reconocidamente difícil, compleja y analítica. La siguiente sección describe una serie de programas de enseñanza diseñados para hacer explícitos algunos de los aspectos epistemológicos fundamentales de la mecánica newtoniana.

\section{PROGRAMAS SOBRE FUERZA Y MO- VIMIENTO}

\section{Introducción}

Se diseñó una serie de programas de ordenador para ayudar a los estudiantes que tenían concepciones tipoimpetus a que cambiaran a las concepciones newtonianas de fuerza y movimiento. La estrategia en que se basaba el programa consistía en tres pasos: primero, establecer criterios para decidir qué es y qué no es una fuerza; segundo, usar estos criterios para demostrar que hay problemas con la idea del movimiento como fuerza; y tercero identificar dicha "fuerza/movimien. to" con la cantidad de movimiento que se interpreta como el resultado del efecto de fuerzas que han actuado previamente.

Existen características de los programas dignas de ser resaltadas. En primer lugar, fue necesario poder discutir una fuerza prototipo sobre la que hay consenso general. Los prototipos más comunes son los empujones y estirones humanos, pero tienen el problema de que son generalmente de magnitud variable y duración Limitada. La fuerza ejercida por un cohete, en cambio, es claramente una fuerza activa. También es fácil imaginar un cohete ejerciendo una fuerza constante; y pensar que uno podría usar un botón para controlarla. Una característica común de los programas es, por consiguiente, el uso de cohetes para suministrar muchas de las fuerzas que se consideran.

Una segunda característica notable es que se incluye de modo explícito la relacion entre causa y efecto. La in. clusión de la relación causa/efecto (RCE) es necesaria porque forma una parte esencial de los criterios que usan los estudiantes para decidir si está actuando o no una fuerza. Los programas están basados en el supuesto de que la relación causa/efecto en su forma lógica más simple será comprendida y aceptada por los estudiantes. En otras palabras, se ha supuesto que los estudiantes que usan estos programas aceptan que los hechos físicos ocurren como un resultado de la acción de algún agente causal, y que no ocurren por sí mismos. No obstante, los programas también están basados en la suposición de que no todos los estudiantes comprenderán las implicaciones detalladas de la aplicación de la relación causa/efecto (RCE) al caso específico de la fuerza y el movimiento. Más concretamente, el programa plantea las preguntas:

a. ¿Qué debe considerarse como (uña) causa?

b. ¿Qué debe considerarse como (un) efecto?

c. ¿Cuál es la naturaleza de la relación entre causa y efecto?
Al contestar estas cuestiones, es significativo que el papel jugado por la RCE tiene que cambiar de ser inicialmente subsidiario de la experiencia cotidiana a llegar a ser parte de la base para reinterpretar los fenómenos en un modo que contradice la experiencia cotidiana. El contenido de la enseñanza se suministra mediante una secuencia de programas. Puesto que la Iongitud de Ios programas viene determinada por consideraciones de programación más que de contenido, en lo que sigue, se considerarán unidades temáticas de contenido en lugar de programas.

Los tópicos se resumen en una serie de tablas que contienen ejemplos de fuerza y movimiento, comentarios sobre la física y los argumentos causa-efecto de los ejemplos, y obtención de conclusiones. La notación usada en los ejemplos incluye $i$ : el estado inicial de mo vimiento del módulo, $a$ : la acción tomada para influir en el movimiento, $y f$ : el estado final de movimiento del módulo.

\section{A. Tópico I: Dada una fuerza, hallar el movimiento.}

El propósito de este tópico es considerar cómo el movimiento de un módulo propulsado por cohetes es afectado por la fuerza que un motor de un sólo cohete ejerce sobre el módulo, planteando dos preguntas:

a) ¿Qué efecto tiene una fuerza constante (que actúa durante un período constante de tiempo) sobre el movimiento del módulo?

b) ¿Cómo afecta la velocidad inicial del módulo a esta respuesta?

El movimiento del módulo se simula en la pantalla, a lo largo de una trayectoria horizontal. Debajo de la trayectoria hay una escala de velocidad, que marca la velocidad mediante una aguja móvil. Se puede, por tanto, ver la velocidad como el movimiento del módulo y registrada en el velocímetro. Cuando el módulo alcanza el extremo de la pantalla desaparece y aparece en el lado opuesto con la misma velocidad.

Se consideran las dos preguntas anteriores en una serie de ejemplos:

1.a. La velocidad inicial $v_{i}$ es cero.

l.b. La $v_{i}$ es distinta de cero en la misma dirección y sentido que la fuerza.

I.c. La $v_{j}$ es distinta de cero en sentido opuesto a la fuerza y lo suficientemente grande para que el módulo aminore la marcha sin cambiar de sentido.

I.d. La $v_{i}$ es distinta de cero en sentido opuesto a la fuerza y lo suficientemente pequeña para que el módulo cambie de sentido.

En cada caso, después del primer ejemplo, se da la oportunidad al estudiante de mirar el movimiento y predecir la velocidad fínal $v_{f}$ del módulo antes de que el resultado se muestre en la escala. Las predicciones incorrectas llevan a bucles de retroalimentación. Todas 
Tabla I

Resumen del Tópico I: Dada ura fuerza, hallar el movimiento.

i: estado inicial, a: acción realizada, f: estado final

\begin{tabular}{|c|c|c|}
\hline PrgPtSizo & EJEAPLOS & EISICA \\
\hline $\begin{array}{l}\text { Uso de la experiegciz } \\
\text { tonathente aceptadz l,a }\end{array}$ & $\begin{array}{l}\text { i) adsuio en reposo } \\
\text { 2: appija gstandars (EE) + }\end{array}$ & $\begin{array}{l}\text { Is tuerza (enpuje) tabia } \\
\text { peposo en woyinianto }\end{array}$ \\
\hline \multicolumn{3}{|l|}{ 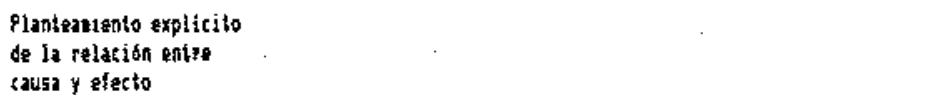 } \\
\hline 1.6 & 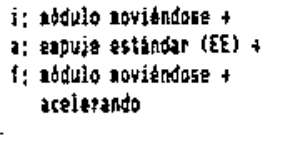 & $\begin{array}{l}\text { Ia fubra (eupuje) cabia } \\
\text { la velosidad aluentanto } \\
\text { su menitud }\end{array}$ \\
\hline 1,6 & 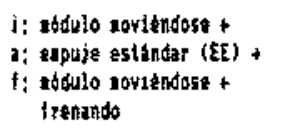 & $\begin{array}{l}\text { Ia fuetza (eapuje) tabia } \\
\text { la velosidad disusmiyanto } \\
\text { sy angnitud }\end{array}$ \\
\hline 1.6 & 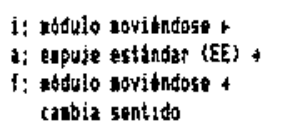 & $\begin{array}{l}\text { Ia fuerze teupuje) canbia } \\
\text { ia velotidad inyirs ionto } \\
\text { su sentido }\end{array}$ \\
\hline
\end{tabular}

Argunento para estabiezer que significa "el nisao of

Dbtencton de conclusiones
Une tuem protuse un canbio de velocidad (en uagtisus y/o sentido)

La nisnz fuerta (EE) produse el tisso tabio de voloridad, tndopandianteante de la yelociotod inicias
CALSALIDAO

causz (fuerza) grodute effecto (reposos adyitiento)

\author{
Iz cicas causa (fustza o \\ EE) actúa en a, $b, c, d$ \\ iptodan considomarse cono \\ alastos tgules: \\ reposo + corlsianto \\ auats to te magnitud \\ discinution to sagnitud \\ inversito de sentido? \\ si al stacto as cabbio de \\ valocidas, entonces se \\ grotute al bicaso of asto \\ in $\left.a_{1}, b_{1}, c_{1}\right\}$ \\ una caust ffuerza) producs \\ un secto tcanbio de \\ velocidad) \\ La sich soust (funk \\ tel EE) produce ol sisto \\ fecto (tanabio on reloctdad?
}

las alternativas están diseñadas para conducir a las siguientes respuestas a las anteriores cuestiones:

a.Una fuerza cambia el movimiento del módulo, i.e., cambia la magnitud y/o el sentido de la velocidad del movimiento.

b.EI cambio de movimiento no depende de la velocidad inicial del módulo, i.e., el cambio de movimiento será el mismo para cualquier velocidad inicial.

Las ideas de causa y efecto se introducen en el ejemplo I.a., con una concepción limitada de su significado: el empuje de un sólo cohete durante un intervalo de tiempo dado es la causa, y el movimiento (contrapuesto sólo al no movimiento, al reposo) es el efecto. Es de esperar que esta concepción sea comúnmente aceptada, porque el empuje del cohete es, sin desacuerdos, un empuje activo que puede convertir el reposo en movimiento. Los ejemplos I.b., I.c. y I.d. mantienen la causa invariable (la misma fuerza durante el mismo tiempo), permitiendo así cuestionarse qué debe considerarse como efecto, y abordar la relación entre causa y efecto. Esto se hace planteando que la $R C E$ requiere que la misma causa debe producir el mismo efecto, y considerando entonces diferentes posibilidades de lo que debería considerarse como "el mismo efecto". Más concretamente, cuando la velocidad inicial varía, ¿podría significar el "mismo efecto" la misma velocidad finai, o el mismo cambio de velocidad, o no debe esperarse hallar el mismo efecto bajo estas condiciones? Las simulaciones muestran que la misma fuerza produce el mismo efecto, con tal que éste sea interpretado como el mismo cambio de velocidad.

La Tabla I resume el tópico I. 


\section{B. Tópico II: Sin actuar fuerza alguna, hallar el movimiento.}

El propósito de este tópico es mirar el mismo problema de una manera ligeramente diferente, planteando la cuestión:

¿Qué ocurre al movimiento del módulo cuando no hay fuerza actuando sobre él?

La cuestión se enfoca considerando que el módulo está en ei espacio exterior, lejos de cualquier influencia posible. Al comienzo se establece que no hay fuerza actuando sobre el módulo. Se consideran dos ejemplo separados:

\section{II.a. El módulo está inicialmente en reposo.}

II.b. El móduio está moviéndose.

Se le pide en cada caso al estudiante que prediga si la velocidad inicial será la misma o cambiará. La respues ta se obtiene de dos maneras, por simulación en la pantalla, y siguiendo un argumento basado en la negación del resultado del tópico I: si no hay fuerza no puede haber cambio de movimiento.

Con respecto a la RCE, este tópico se edifica sobre los resultados previos. Se aborda la RCE preguntando si "la misma causa significa el mismo efecto" implica "ausencia de causa significa ausencia de efecto". Concretamente, la implicación se confirma mostrando que cuando no actúa fuerza alguna, no hay cambio en la velocidad.

La Tabla II resume el tópico II.

\section{Tópico III: Dadas más de una fuerza, hallar el movimiento.}

El propósito de este tópico es generalizar el concepto de fuerza resultante. Se empieza planteando la cuestión:

¿Qué efecto tendrán dos o más fuerzas sobre el movimiento del módulo?

Se usa un módulo más complicado. Tiene cuatro cohetes, cada uno de los cuales es capaz de ejercer una fuerza de igual magnitud, dos actuando hacia la izquierda y dos hacia la derecha. El movimiento del módulo se simula como antes, y su velocidad se muestra en un marcador digital en vez de como una flecha moviéndose en una escala de velocidad. Se presentan las siguientes situaciones:

III.a. Un cohete encendido (para calibrar la magnitud de su efecto).

\begin{tabular}{|c|c|c|c|}
\hline PrOPBSSITO & EJEXPLQS & GASIEA & CANSALLABAO \\
\hline \multirow[t]{2}{*}{$\begin{array}{l}\text { Hagacion de is causa lla } \\
\text { signalista negacion del } \\
\text { teacto }\end{array}$} & 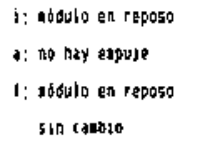 & $\begin{array}{l}\text { \{yerza nula (eopuse nulo) } \\
\text { groduca un cantio nulo } \\
\text { dy velotidad }\end{array}$ & 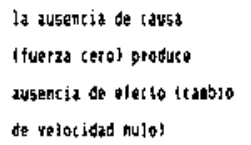 \\
\hline & 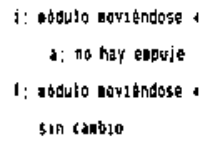 & $\begin{array}{l}\text { thetra nula (enpuse nulo) } \\
\text { produce un cantio nulo } \\
\text { of velociose }\end{array}$ & 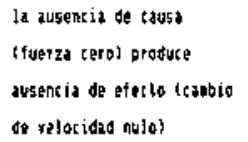 \\
\hline Obtencion se conclusiones & & 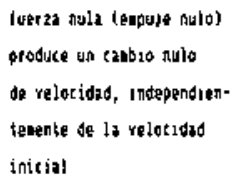 & 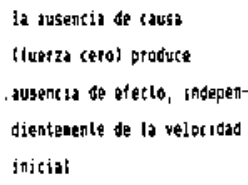 \\
\hline
\end{tabular}


Tabla III

Resumen del Tópico III: Dadas más de una fuerza, hallar el movimiento. i: estado inicial, a: accion realizada, f: estado final.

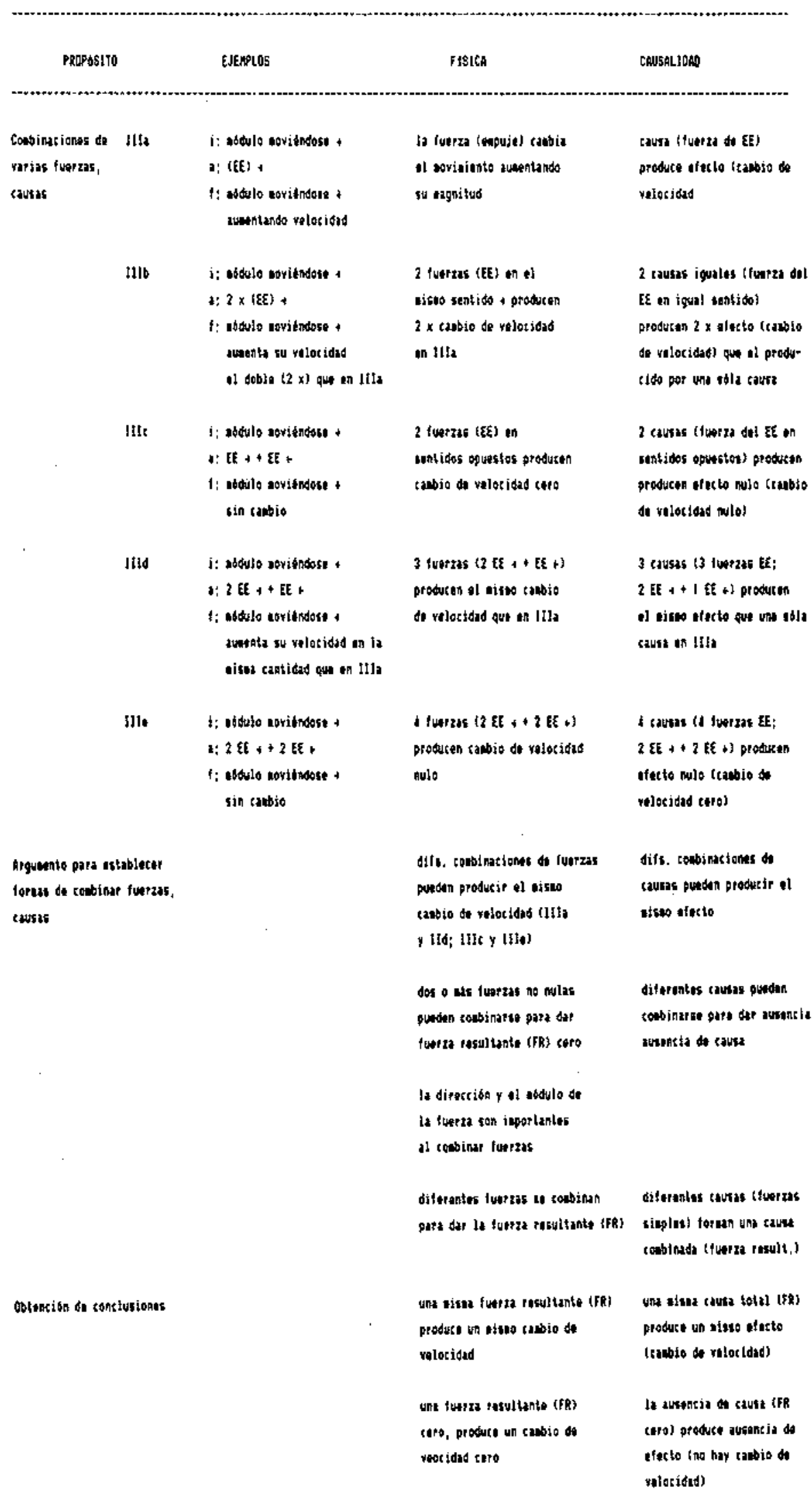


III.b. Dos cohetes encendidos en el mismo sentido.

III.c. Dos cohetes encendidos en sentidos opuestos.

III.d. Tres cohetes encendidos, uno hacia la izquierda y dos hacia la derecha.

III.e. Cuatro cohetes encendidos, dos a la izquierda y dos a la derecha.

En cada caso a partir del primero, se le pide al estudiante que prediga la velocidad final, $y$ al final que intente dar una respuesta a la cuestión, con sus propias palabras.

Ésta se resume como:

a. El módulo se mueve como si una sola fuerza estuviera actuando sobre él (la fuerza resultante), i.e., la fuerza resultante produce los mismos efectos señalados en los tópicos I y II.

b. La fuerza resultante se obtiene combinando todas las fuerzas que actúan sobre el objeto.

c. Al combinar las fuerzas es necesario tener en cuenta tanto el sentido como la magnitud de cada fuerza.

d. Al hacer esto, dos o más fuerzas no nulas pueden combinarse para dar una fuerza resultante nula, i.e., una resultante de magnitud cero, o inexistencia de fuerza.
Con respecto a la $\mathrm{RCE}$, las series de ejemplos usando configuraciones diferentes de fuerzas distintas conducen a una definición más general de la causa del movimiento, a saber, una fuerza resultante, obtenida por combinación de fuerzas individuales usando reglas específicas. En este caso, el requerimiento de la RCE de que el mismo efecto sea producido por la misma causa pone restricciones a las reglas de combinación.

El tópico III se encuentra recogido en la Tabla IrI.

\section{Tópico IV: Dadas las fuerzas, hallar el movimiento.}

Los propósitos de este tópico son, en primer lugar, combinar las dos generalizaciones más importantes consideradas en los tópicos anteriores, es decir, la independencia de los cambios de movimiento de la velocidad inicial, y la combinación de fuerzas en una fuerza resultante; y en segundo lugar, resumir los ejemplos anteriores en términos de causa y efecto, estableciendo una comparación explícita entre "dada una fuerza, hallar el movimiento" y "dada una causa, hallar el efecto". En lo que se refiere a los ejemplos considerados, "causa" es interpretado como "fuerza resultante", "efecto" es interpretado como "cambio de velocidad" $y$ se muestra la naturaleza de la RCE para incluir su negación, "no causa significa no efecto", y su uso para predecir, "dada la causa, hallar el efecto".

El tópico IV se resume en la Tabla IV.

PFOPGSITA FISICA CAUSALIDAO

\section{Contestar al problena}

básico conbinar diferentes fuerzas

parz obtener la fuerza resultante

Ia fuerza resultante (FR)

attuando sobre un cuerpo

produre canbio de velocidad

si Fh es caro, entonces no hay

canbio de velociload conbiant causas individuales

para producir una causa conbinada causa (FR) produce afecto

(canbio de valocidad)

Si no bay causs, no hay

stesto 


\section{E. Tópico V: Dado el movimiento, hallar la fuerza.}

El propósito de este tópico es empezar una exploración inicial de las implicaciones del problema: "dado el mo. vimiento, hallar la fuerza"; obtenido dando la vuelta al problema previo: "dada la fuerza, hallar el movimien" to". El énfasis principal en esta fase está en usar el problema para decidir qué magnitudes pueden ser consideradas fuerzas.

Este tópico explora la RCE más allá, al considerarla en forma inversa: "dado el efecto, encontrar la causa". Al hacer esto, la RCE está siendo usada en un nuevo papel, el de definir que puede ser considerado como causa. Por consiguiente suministra un criterio -interno a la teoría de la dinámica - de lo que puede y no puede considerarse como fuerza. Esto es expresado como: $\mathbf{S i}$ algo produce el mismo efecto que el de una fuerza reco- nocida (tal como el empuje de un cohete), entonces es una fuerza.

Si no puede producir el mismo efecto, entonces no es una fuerza.

Este tópico se resume en la tabla V.

\section{F. Tópico VI: ¿Es el movimiento una fuerza?}

EI propósito de este tópico es considerar si el movimiento continuo de un objeto después de que una fuerza haya dejado de actuar debe ser considerado una fuerza. Esto es particularmente relevante, ya que muchos estudiantes se refieren a éste como la "fuerza del movimiento", la "fuerza del objeto", o la "fuerza del (agente original)" (incluso aunque haya cesado de actuar). El

\begin{tabular}{|c|c|c|}
\hline PROPASIFO & FtSICA & CAUSALIOAD \\
\hline $\begin{array}{l}\text { Planteniento del probleas } \\
\text { Disito thverso }\end{array}$ & $\begin{array}{l}\text { si bay canbio de velocidad, } \\
\text { dabe astar actuando una FR }\end{array}$ & $\begin{array}{l}\text { si hay un stecto (tanbio } \\
\text { de velocidad), entoneas dabe habar habtdo } \\
\text { una causa (fuerta resultante) }\end{array}$ \\
\hline
\end{tabular}

si no hay tantio of veloridad, antontes la FR dabe ser caro

\author{
si no hay efecto, \\ no puedo haber sausa
}

\begin{abstract}
asunto se aborda de dos maneras, primero, considerando un argumento basado en la suposición de que el movimiento es una fuerza, y segundo, aplicando el criterio del tópico $\mathrm{V}$.
\end{abstract}

Para estudiantes que plantean que hay una "fuerza/ movimiento", se consideran una serie de ejemplos:

VI.a. Ejemplo II.b: Un módulo moviéndose en el es. pacio exterior con velocidad constante. Esto se usa para clarificar su comprensión de la "fuerza/movimiento", a saber, que tiene el mismo sentido que la velocidad, y aumenta con ella.

VI.b. Ejemplo I.b: Un módulo acelerando bajo el empuje de un cohete, aunque todavía en el espacio exterior.

VI.c. Ejemplo I.c: Un módulo frenando bajo el empuje contrario de un cohete.
EI argumento se apoya en el concepto de fuerza resultante, y usa los hechos de que, primero, el sentido de la fuerza resultante del empuje y de la "fuerza del movimiento" depende de sus magnitudes y sentidos relativos (del tópico III), y, segundo, la magnitud de la "fuerza del movimiento" aumenta con la velocidad del objeto (del ejemplo VI.a.). El objetivo del ejemplo VI.b. es mostrar que la fuerza resultante del empuje y de la "fuerza del movimiento" en el mismo sentido que la velocidad, produce aceleración. Esto tiene la intención de hacer el concepto de fuerza resultante más plausible. Entonces, en el ejemplo VI.c., al considerar un objeto que se está moviendo lo suficientemente rápido, es siempre posible tener una "fuerza de movi" miento" mayor que un empuje constante, y por tanto tener una fuerza resultante en el sentido del movimiento de un objeto que está aminorando la marcha. Esto contradice el resultado del tópico III, basado en la acción de fuerzas prototipo, creando así la duda sobre 
Estabiecer propiedades

de lo tuerza/aoviniento

Ma

i: sdulo aovikndose +

a) Eupsise nulo

$f$ a nodulo moviendose +

sin caabio

Mostrar ia contribucion, , vib

i) nduito noviendose +

de la fuerza/zoviniento

a la fuarza resutante

a: elpuje estándar +

$f:$ addulo ariencose +

ausentando velocidad

Hostraf contradiciones Wit

i: ndulo novitnosese +

entre los sentidos de la

fuerza regultante dgterninados

por dos vétodos

a: $\mathrm{EE}+$

li sdulo coviéndose

trenando
Hozitar que Fin no puade

ser una fuerza

Mostrar que fry es

coutentsa: aje al afecto

as sabio de wonentua avviniento con relocidad constante;

la fuerza/roviniento (FM) en el sentido

de la velocidadi el ádulo aunenta

ton la veloridad

aoviaiento acelerado

(avnentando yelocidad):

$F R+=E H+E E+$

moviniento acelerado (trenanco);

por tanto, del Topico III, fâ

debe estar en sentido t; pero FH $t$; $E$ E + ,

$\mathrm{FR}$ ? = FH + EE +, si la velocioad

aukenta suficientesente, la $F 8$ irs $t$,

por fanto, contradicsion

si fla no produce un canbio

5i algo no produse

te velocidad, no puede

un efecto, no ptiede

ser tha fuesza

set unz causa

euando astua s\$lo $\mathrm{FH}_{\mathrm{f}}$ la

velocidad peruanece constante

ten vla); puzsto gue no

anbia is veloridad, fll no

pusede ser ura fuerza

Fal tanbia cuando una fuerza

el efacto es canbio of Fir:

(EE) attú (VIb, WIs); F⿱艹 cuando hay una cassa, hay constante cuando no astua ninguna fuerza (VIa); por tanto un efecto; cuando no hay catse, no hay ofecto puesto que na habia efecto (cabio de velocidad), no habia una caust (ftuerz resul tante)

Fil esta reiacionata con la

relocidad; es comentuk 
la suposición de que la "fuerza/movimiento" es una fuerza.

En segundo lugar, se usa el criterio del tópico V para considerar si una "fuerza/movimimiento" por sí misma puede aumentar la velocidad de un objeto, o cambiar su dirección de movimiento. Puesto que es incapaz de hacer esto, no puede ser considerada en la misma categoría que una fuerza prototipo. Por último, se considera el punto de que si bien la "fuerza/movimiento" no puede ser considerada como una fuerza, es real, es decir, hay una diferencia clara entre un objeto en reposo y uno que se está moviendo. La "fuerza/movimiento" se identifica como cantidad de movimiento (momentum). Se generaliza el efecto para cambios de momentum. El momentum existente es el resultado de un efecto causado por una o más fuerzas que ya no actúan.

La RCE se aplica en este tópico de dos maneras. Primero, el tópico asume que el movimiento es una fuerza, incorporándola como parte de la fuerza resultante, o causa, y utiliza la RCE para predecir el movimiento futuro, como en el tópico IV. La predicción es contradicha por una experiencia simulada, y el tópico sugiere que el modo de salir de la contradicción es renunciar a la suposición. Segundo, este tópico muestra que el movimiento no cumple el criterio del tópico $\mathrm{V}$, y por tanto no puede ser considerado como una causa. Un breve análisis muestra que puede, no obstante, ser considerado como el resultado de alguna causa primera.

La tabla VI resume este tópico VI.

\section{DISCUSIÓN DE LOS PROGRAMAS}

Los programas fueron diseñados teniendo en mente varias consideraciones. Éstas incluyen:

- El contenido físico que se enseña, y las interpretaciones y dificultades de los estudiantes con el contenido.

- Los aspectos epistemológicos en los que se fundamenta la física.

- Una concepción del aprendizaje del estudiante como cambio conceptual.

Después de que consideremos estos aspectos, compararemos los programas con los puntos surgidos en la revisión de trabajos sobre enseñanza realizada anteriormente.

\section{A. EI contenido físico de los programas}

El contenido físico de estos programas es, siempre que ha sido posible, cualitativo, los ejemplos son todos unidimensionales, y la discusión general de la dinámi- ca incorporada en estos programas está diseñada para preceder al análisis detallado cuantitativo de Ia cinemática con la que empiezan la mayoría de los cursos introductorios de física. Hubo distintas razones que guiaron esta elección.

Por una parte, los programas se diseñaron para enfrentarse a algunos de los aspectos que han sido identificados por la inyestigación en las concepciones de los estudiantes. Éstas incluyen una confusión entre los conceptos de velocidad y aceleración, un uso generalizado de ideas tipo-impetus como agentes causales, $e$ inconsistencia en el uso de ideas teóricas. Se trata de aspectos físicos, cualitativos. Por tanto, los programas se diseñaron para afrontar la esencia de la física, más que Ias complejidades de las matemáticas usadas para representar la física. Esto no supone negar que las matemáticas son un requisito esencial para poder hacer física, sino reconocer que en sí mismas no son suficientes. Las deficiencias en matemáticas son bastante más aparentes que las de comprensión física, probablemente porque las matemáticas, para hallar la componente $x$ de un vector, o para determinar la aceleración a partir de una gráfica velocidad/tiempo, se hacen explícitas en la solución de probiemas, en mucho mayor grado que la comprensión de por qué es importante centrarse en la aceleración y no en la velocidad, o de cuáles fuerzas deberían incluirse para calcular la fuerza resultante. Por tanto, con el fin de centrarse en la esencia física de la fuerza y el movimiento, se eligió el contenido para reducir las matemáticas requeridas.

En segundo lugar, los programas se basaron en la suposición de que si los estudiantes saben por qué es necesario hacer algo que es difícil y complicado, es más probable que lo entiendan. Concretamente, a la luz de las investigaciones que muestran que muchos estudiantes no discriminan entre velocidad y aceleración, parece necesario comprender la importante diferencia entre las dos y por qué la aceleración juega un papel fundamental en la mecánica newtoniana que la velocidad. Un objetivo importante de los programas fue mostrar que dicha comprensión surge a partir de la dinámica cualitativa, es decir, que el cambio de velocidad (el ingrediente esencial de la aceleración), y no la velocidad misma, es el efecto causado por la acción de fuerzas.

No pretendemos que la física de los programas esté ordenada según Ia mejor de las secuencias posibles, o que sea completa. Con respecto a la secuenciación, la necesidad de contemplar un número de aspectos diferentes usando un abanico de ejemplos parece ser más importante que la secuencia particular, especialmente porque las diferencias individuales, probablemente, hagan que sean adecuadas secuencias distintas para diferentes estudiantes. Esencialmente, esto significa que los programas de este tipo deben ser flexibles. Con respecto a si son completos, hay muchas extensiones que necesitan contemplarse. Algunas de éstas podrían ser la generalización a objetos de masa diferente, a fuerzas que actúan durante tiempos distintos, fuerzas variables, y la consideración de la rapidez de cambio de la velocidad. Otras extensiones importantes podrían ser movimientos en dos y tres dimensiones. 


\section{B. Aspectos epistemológicos}

El tema causa-efecto constituye un elemento esencial de los programas. No obstante, lo que es particularmente significativo es Ia manera en que cambia su papel. El primer ejemplo del tópico I recurre a la experiencia común de que cuando un agente activo de suficiente magnitud, tal como el empuje de un cohete, actúa sobre un objeto en reposo, hace que éste se mueva. El ejemplo usa un caso prototipo de fuerza, y caracteriza el movimiento sólo en contraposición al no movimiento. En otras palabras, los términos "fuerza" y "movimiento" se obtienen de la experiencia. Las relaciones causaefecto (RCE) son así claramente subsidiarias de la experiencia, y sus significados son demasiado ambiguos para que sean considerados como definiciones.

En este punto, la implicación de la RCE según la cual "Ia misma causa conduce al mismo efecto" se plantea explícitamente, y se aplica en los ejemplos $2-4$ del tópico I con una fuerza constante, pero distintas condiciones iniciales. Esto impone una restricción sobre lo que puede ser considerado como efecto que conduce a una definición operativa de "efecto" que es más específica que la diferencia entre movimiento y no movimiento. En otras palabras, Ia RCE está sirviendo ahora como guía para la observación de la experiencia. En el tópico III, se usa de nuevo la definición operativa de "efecto" para clarificar el significado de "fuerza" cuando está actuando más de un agente activo, conduciendo, por tanto, a una definición de "causa" como "fuerza resultante". La RCE puede usarse ahora para decidir qué puede y qué no puede ser considerado como fuerza. En el tópico IV esto lleva a la conclusión de que el "movimiento" no es una fuerza, en contradicción con la ampliamente mantenida idea intuitiva del impetus como fuerza. La RCE ha invertido así su papel, y cobra predominio sobre la experiencia. La importancia de ello es que dicho papel parece ser esencial para cualquier teoría que persiga realizar predicciones con éxito.

Los presupuestos epistemológicos de generalizabilidad y consistencia interna también juegan papeles esenciales en la secuencia de programas. Un ejemplo de ello se encuentra en el tópico I, en el cual se aplica la misma fuerza al módulo bajo condiciones iniciales distintas. Implícito en la búsqueda del "mismo efecto" hay un presupuesto de generalizabilidad, i. e., de la importancia de buscar un modelo, una tendencia que vaya más allá de los detalles específicos de cada ejemplo. Para un estudiante que no sostenga este presupuesto (compromiso), la conclusión de que puede identificarse un efecto común podría no tener sentido. Un ejemplo del papel de la consistencia interna se da en el tópico VI. Al aplicar consistentemente el concepto de "fuerza resultante" del tópico III a la "fuerza/movimiento" (que según los estudiantes es responsable de que continúe el movimiento) y a una fuerza opuesta producida por el cohete se llega a una predicción que es contradictoria con la experiencia. Sin un presupuesto de que una teoría debe tener consistencia interna, un estudiante con una concepción "fuerza/movimiento" podría no aceptar la predicción , y no encontrar pues el resultado contradictorio.

\section{Aprendizaje como cambio conceptual}

La idea de que el aprendizaje puede ser considerado como un cambio en una concepción del estudiante ha sido discutida en otros sitios $(2,24)$. Esta concepción sugiere que determinar las concepciones que tienen los estudiantes sobre un tópico particular, comprender por qué las tienen, e identificar las condiciones bajo las cuales las cambiarán, son aspectos muy importantes para el diseño de la enseñanza. Esto, a su vez, sugiere que los profesores deberían usar estrategias que permitan diagnosticar las concepciones de los estudiantes, que permitan disminuir el estatus de las concepciones que probablemente obstaculicen el aprendizaje del contenido deseado, y que aumenten el estatus de las nuevas ideas.

El propósito principal de los programas descritos anteriormente era la segunda de estas estrategias, la disminución del estatus, en este caso, de la concepción de que el impetus o movimiento es una fuerza que hace que el movimiento continúe. Los programas no se diseñaron para ser usados aisladamente, sino con alguna forma de evaluación diagnóstica tales como los cuestionarios usados en los trabajos de investigación que se hallan en la literatura, o con programas adicionales para el diagnóstico.

Para reducir el estatus de una concepción, es necesario mostrar que hay razones para estar insatisfecho con ella. A su vez, esto requiere que la concepción se evalúe sobre una base que sea aceptada por el estudiante y que muestre claramente por qué hay problemas con la concepción. ¿Por qué, entonces, una concepción típoimpetus es inaceptable en un curso introductorio de física? Después de todo, surninistra una explicación de por qué los objetos, e. g., una pelota de golf, se mantiene moviéndose bastante después de que agentes obvios hayan actuado sobre ellos. No obstante, esto presenta algunos problemas evidentes desde la perspectiva de la investigación científica. No predice el movimiento futuro con precisión. No es una teoría autoconsistente. No existen criterios claros sobre cuando debe o no debe usarse. Se trata, en otras palabras, de una "teoría reactiva", es decir, da explicaciones a posteriori de una serie de fenómenos que ocurren naturalmente, pero precisamente porque es ad hoc, amorfa, poco-definida, y su causalidad es subsidiaria de la experiencia, no es útil como teoría predictiva.

Esto suministra una posible explicación de la persistencia de las concepciones tipo-impetus entre los estudiantes que han seguido cursos de mecánica newtoniana, y sugiere un remedio. Si dichos estudiantes no tienen una comprensión adecuada de la naturaleza y la potencia de una teoría pređictiva, no tendrán bases para estar insatisfechos con sus propias ideas. Un objetivo importante de los programas fue, por tanto, centrarse explícitamente sobre el significado y función de la relación causa-efecto, para aplicarla al contenido físico en cada fase, y usarla, así, como una base adecuada para crear insatisfacción con sus concepciones tipoimpetus. El papel esencial de la RCE se muestra en el análisis de los presupuestos epistemológicos presenta- 
do anteriomente, que muestra que a menos que un estudiante crea que su teoría tiene que ser internamente consistente, generalizable, y fuertemente causal, la estrategia incorporada en los programas no puede funcionar.

Al abordar de esta manera las concepciones tipo-impetus surge un beneficio adicional. La misma base puede ser utilizada para evaluar tanto las concepciones del impetus como las newtonianas. El mismo proceder que conduce a la disminución del estatus de una concepción conduce al aumento de estatus de la otra.

\section{Comparación con los estudios sobre enseñanza}

Los estudios de instrucción revisados previamente incluían el suministro de actividades con el fin de permitir que los alumnos:

*Se plantearan ejemplos distintos.

* Identificaran y expresaran sus concepciones actuales verbalmente o respondiendo a un movimiento simulado de una u otra manera.

* Exploraran las implicaciones de sus concepciones en un conjunto de situaciones, que les requirian explicaciones y predicciones autoconsistentes.

* Advirtieran las limitaciones de las concepciones que sostenían.

En comparación con estos objetivos, los programas sobre fuerza y movimiento suministraron diferentes ejemplos, aunque centrados en el denominador común de un módulo impulsado por cohetes; no diagnosticaron explícitamente las concepciones de los estudiantes, estando diseñados para ser usados junto con otros medios de diagnóstico; exploraron el contenido a través de una serie de situaciones diferentes cuya interrelación fue considerada frecuentemente para buscar consistencia global; y su propósito principal era hacer que los estudiantes estuvieran insatisfechos con las concepciones tipo-inpetus que mantenían.

La implicación activa de los estudiantes es esencial para todos estos puntos. Cambiar el modo de pensar propio es un proceso activo, y requiere una consideración seria y reflexiva de los distintos aspectos. Una de las funciones de la discusión en clase es fomentar este tipo de implicación, pero como varios estudios han mostrado, esto podría alcanzarse mediante programas de ordenador diseñados apropiadamente. Queda por ver si esto continuará siendo así con programas, como los usados aquí, con gran componente didáctico.

\section{CONCLUSIONES}

Al abordar el problema de los estudiantes que mantienen tenazmente concepciones del movimiento tipoimpetus y los factores que influyen en él, el modelo de cambio conceptual sugiere que hay ciertas cosas que es necesario conocer. Se trata no sólo de las ideas que tienen los estudiantes sino también de comprender por qué las tienen. Más específicamente, se necesita saber por qué no ven los inconvenientes de una concepción tipo-impetus, y por qué no parecen ver las ventajas de las concepciones newtonianas.

Una revisión de la literatura sugiere que las concepciones tipo-impetus de los estudiantes son ideas que son una interpretación simple de la experiencia, y que no están basadas en una comprensión precisa de causa y efecto. En otras palabras, estas ideas están basadas en una epistemología débil. Por el contrario, la mecánica newtoniana está basada en una fuerte relación causaefecto, cuya aplicación conduce a conclusiones no intuitivas. Esto quiere decir que hasta que un estudiante no advierta la potencia de una relación causa-efecto fuerte, y aprenda a aplicarla consistentemente, no es probable que acepte las ideas newtonianas.

Los programas sobre fuerza y movimiento se diseñaron para integrar conceptos de cinemática y dinámica con la enseñanza explícita de la epistemología subyacente. Aunque una evaluación de la capacidad de los programas para ayudar al cambio conceptual debe basarse en proebas empíricas, la inclusión explícita de la epistemología de una relación estricta causa-efecto parece ser necesaria. Sin ella, parece no existir una base para que los estudiantes hagan cambios racionales en sus concepciones de fuerza y movimiento.

\section{REFERENCIAS BIBLIOGRÁFICAS}

CARAMAZZA, A., MCCLOSKEY, M. y GREEN, B., 1981. Naive beliefs in "sophisticated" subjects: Misconceptions about trajectories of objects, Cogn., 2, pp.117-123.

CHAMPAGNE, A.B., KLOPFER, L.E. y ANDERSON, J.H., 1980. Factors influencing the learning of classical mechanics, Am. J. Phys., 48, pp.1074-1079.
CHAMPAGNE, A.B., GUNSTONE, R.F. y KLOPFER, L.E., 1985. Instructional consequences of students" knowledge about physical phenomena. Effecting changes in cognitive structures among physics students, in West, L.H.T. y Pines, A. (eds.), Cognitive structure and conceptual change, (Orlando, F.L.: Academic Press), pp. 61-90 y pp. 163 187 . 